\title{
Changes and clinical significance of serum inflammatory factors in the treatment of pregnancy hypertension syndrome with magnesium sulfate combined with nifedipine
}

\author{
FEIFEI ZHAO, FANG AI, JIE WU and XUDONG DONG
}

\author{
Obstetrics Department, The First People's Hospital of Yunnan Province (The Affiliated Hospital \\ of Kunming University of Science and Technology), Kunming, Yunnan 650032, P.R. China
}

Received October 15, 2019; Accepted April 14, 2020

DOI: $10.3892 /$ etm.2020.8863

\begin{abstract}
Curative effect of magnesium sulfate combined with nifedipine on pregnancy-induced hypertension and the effect of serum inflammatory factors were investigated. A total of 188 cases of patients were collected as the research subjects. They all had pregnancy-induced hypertension, and were admitted to The First People's Hospital of Yunnan Province hospital from June 2016 to February 2018. There were, 94 patients treated with magnesium sulfate in the control group, and further 94 patients treated with magnesium sulfate combined with nifedipine in the study group. ELISA was used to detect the expression levels of suppressors of cytokine signaling-3 (SOCS-3), interleukin-10 (IL-10) and interleukin 18 (IL-18), and the relationship between serum inflammatory factors and efficacy was analyzed. The curative effect and eutocia rate in the study group were significantly higher than those in the control group $(\mathrm{P}<0.05)$, and the cesarean section rate was lower than that of the control group $(\mathrm{P}<0.05)$. In the study group, the adverse reactions were significantly lower than those in the control group $(\mathrm{P}<0.05)$. After treatment, the expression levels of serum SOCS-3 and IL-10 in the study group were significantly higher than those in the control group $(\mathrm{P}<0.05)$, while the expression level of serum IL-18 was significantly lower than that in the control group $(\mathrm{P}<0.05)$. The area under the curve (AUC) of the predictive value of SOCS-3, IL-10 and IL-18 in pregnancy-induced hypertension was $0.717,0.727$ and 0.725 , respectively. The best specificity was $76.19,52.98$ and $61.90 \%$, respectively, when the cut-off value was $<0.553 \mathrm{ng} / 1,48.06 \mathrm{ng} / \mathrm{ml}$ and $269.46 \mathrm{ng} / \mathrm{ml}$, and their sensitivity was $70.00,94.74$ and $85.00 \%$, respectively.
\end{abstract}

Correspondence to: Dr Xudong Dong, Obstetrics Department, The First People's Hospital of Yunnan Province (The Affiliated Hospital of Kunming University of Science and Technology), 157 Jinbi Road, Xishan, Kunming, Yunnan 650032, P.R. China

E-mail: xe283r@163.com

Key words: magnesium sulfate, nifedipine, pregnancy-induced hypertension, serum inflammatory factors
In conclusion, magnesium sulfate combined with nifedipine significantly improved the disease course of pregnancy-induced hypertension. The levels of SOCS-3, IL-10 and IL-18 in patients are correlated with the curative effect of pregnancy-induced hypertension, suggesting that they have important value in the treatment and monitoring of gestational hypertension.

\section{Introduction}

As a common pregnancy-related complication, pregnancy-induced hypertension is one of the main causes of perinatal morbidity and mortality (1). Reports show that the prevalence of pregnancy-induced hypertension is 4.6-13.1\% worldwide, and the neonatal mortality is $9.2 \%$ (2). Studies have shown that pregnancy-induced hypertension not only causes serious complications during the perinatal period, but also increases the incidence of cardiovascular and cerebrovascular diseases, seriously affecting the health and safety of pregnant women $(3,4)$. Moreover, due to the particularity of drug action mechanism when pregnant women and fetuses take drugs (5), it is particularly important to choose a safe and effective drug treatment.

Magnesium sulfate could relieve spasmolysis, diuresis and pain (6). It could also relax the vascular smooth muscle, dilate the spasmolysis peripheral blood vessels, reduce blood pressure, which is suitable for the treatment of patients with moderate and severe pregnancy-induced hypertension when receiving intravenous injection $(7,8)$. Nifedipine is a type of dihydropyridine calcium antagonist, it is applicable to various types of hypertension (9-11) and has a good effect on intractable and severe hypertension. Wen and $\mathrm{Li}$ (12) found that the combined use of the above two drugs had a good effect in treating pregnancy-induced hypertension. A previous study confirmed that inflammation response may be involved in the occurrence and development of hypertension (13). Suppressors of cytokine signaling-3 (SOCS-3) is a cytokine signal inhibitor. It inhibits various inflammatory factors by mediating JAK/STAT cytokine signal transduction pathway, playing a role in inhibiting inflammatory reactions (14). By staining, Wang et al (15) found expression of SOCS-3 in the vascular endothelium of pregnant women with preeclampsia, and the expression of SOCS-3 was lower than that of healthy pregnant women. Interleukins play important roles 
in many processes, including information transmission, activation and regulation of immune cells, activation of mediating cells $\mathrm{T}$ and $\mathrm{B}$, proliferation and differentiation, and inflammatory response (16). Interleukin-10 (IL-10) is a key biomarker for the diagnosis and treatment of adverse preeclampsia, a common complication of pregnancy-induced hypertension (17). Krishnan et al (18) proved that hypertension was closely related to the abnormal expression of interleukin-18 (IL-18).

At present, the therapeutic effect of magnesium sulfate combined with nifedipine on pregnancy-induced hypertension has been confirmed in the clinic, but there are few studies on the related changes of patients' inflammatory factors. We speculated that the changes in inflammatory factors in patients can better assess the clinical efficacy and adverse reactions. In order to confirm our conjecture, this investigation explored the changes of serum inflammatory factors during the treatment of pregnancy-induced hypertension syndrome with magnesium sulfate combined with nifedipine. It is expected that the progress of pregnancy-induced hypertension syndrome will be predicted by the level of inflammatory factors.

\section{Patients and methods}

General data. This study is a prospective non-randomized controlled study. A total of 188 cases were collected as research objects. These patients had pregnancy-induced hypertension, and were admitted to the hospital from June 2016 to February 2018. Among them, 94 cases were treated with magnesium sulfate and were the control group, and further 94 cases of patients were treated with magnesium sulfate with nifedipine and were the study group. The study was approved by the Ethics Committee of The First People's Hospital of Yunnan Province (The Affiliated Hospital of Kunming University of Science and Technology). Signed informed consents were obtained from the patients and/or guardians.

Inclusion and exclusion criteria. Inclusion criteria: patients who met the diagnostic criteria and were diagnosed with pregnancy-induced hypertension; patients received treatment in the hospital; patients aged 18-40 years; patients who cooperated with research; patients or their immediate family member signed the informed consent; patients with complete medical records.

Exclusion criteria: patients with heart, liver, spleen, lung, kidney and other important organs injury; patients combined with mental illness and speech dysfunction; patients with diabetes; patients with lymph node, organ and tissue metastasis or drug allergy.

Therapies. Both groups received routine antihypertensive therapy, and continuous ambulatory blood pressure and vital signs of mothers and infants were monitored. Pregnant women were required to take a left lying position to improve uterine placental blood supply. Control group: patients in the control group received $25 \%$ magnesium sulfate injection $(60 \mathrm{ml})$ (Tianjin KingYork Pharmaceutical Co., Ltd., SFDA approval no. H12020994), and 5\% glucose solution (500 ml) was added for intravenous infusion. Study group: after being given the same intravenous infusion of magnesium sulfate as the control group, patients received nifedipine controlled-release tablet (Shanghai Xiandai Pharmaceutical Co., Ltd., SFDA approval no. H12020994), 3 mg/t.i.d. orally. The treatment in both groups continued for more than half a month. Both groups were treated for seven continuous days.

Curative effect assessment. Evaluation standards were formulated based on reference (19). Marked effect: edema, proteinuria and other clinical symptoms disappeared, blood pressure was $<140 / 90 \mathrm{mmHg}$. Effective: edema, proteinuria and other major clinical symptoms were significantly reduced, with blood pressure of 140-150/90-100 mmHg. Ineffective: clinical symptoms and blood pressure such as edema and proteinuria were not significantly improved compared with before treatment, or even aggravated. Total effectiveness $=$ marked effect + effective. For patients with large thrombus and patients who failed to respond to conservative treatment, lower extremity venous thrombectomy and lower extremity venous ligation were taken into consideration.

Determination of inflammatory factors in serum. Blood samples were collected before treatment (on the day of admission) and after treatment (on the day of delivery). The samples were sent to the clinical laboratory of the hospital for determination in the morning of the same day. Inflammatory indexes included SOCS-3 (USCNK, SEB684Hu-1), IL-10 (FineTest, ESH0009), and IL-18 (FineTest, ECH0039). Blood samples were added with $0.2 \mathrm{ml}$ of $2 \%$ coagulant, centrifuged at $1,505 \mathrm{x} \mathrm{g}, 4^{\circ} \mathrm{C}$ for $30 \mathrm{~min}$, and stored at $-20^{\circ} \mathrm{C}$. Enzyme-linked immunosorbent assay (ELISA) was used for detection, and the procedure was strictly in accordance with the kit instructions.

Observational indexes. Clinical efficacy were observed and compared between the two groups. Delivery and incidence of adverse reactions were compared between the two groups. The levels of serum SOCS-3, IL-10 and IL-18, and the predictive value of SOCS-3, IL-10, and IL-18 for therapeutic effects in the two groups were compared before and after treatment.

Statistical analysis. SPSS 24.0 statistical software (Shanghai Yuchuang Network Technology Co., Ltd.) was used for statistical calculation of all experimental results. Graphpad 8 (Softhead Inc.) was used for drawing all images and for checking the results twice. Enumeration data were all expressed in the form of (rate), and $\chi^{2}$ test was used for comparison between groups. Measurement data were all expressed in the form of (mean $\pm \mathrm{SD}$ ), and t-test was used for comparison between groups. The predictive value was analyzed by ROC curve. $\mathrm{P}<0.050$ was considered statistically significant.

\section{Results}

Comparison of general data. There were no significant differences in age, BMI, gestational age, fertility circumstance, mean arterial pressure, place of residence, ethnicity, smoking history, the degree of edema, proteinuria, drinking history and exercise habits in the study group and the control group ( $\mathrm{P}>0.050)$, indicating that the two groups of patients were comparable (Table I).

Efficacy evaluation and adverse occurrence comparison. The total effective rate of clinical efficacy was $95.74 \%$ in the study 
Table I. Comparison of clinical data [mean \pm SD, n (\%)].

\begin{tabular}{|c|c|c|c|c|}
\hline Characteristics & Research group $(n=94)$ & Control group $(n=94)$ & $\chi^{2}$ or $\mathrm{t}$ & P-value \\
\hline \multirow[t]{2}{*}{ Age (years) } & & & 1.751 & 0.0815 \\
\hline & $29.38 \pm 1.64$ & $28.91 \pm 2.02$ & & \\
\hline \multirow[t]{2}{*}{$\mathrm{BMI}\left(\mathrm{kg} / \mathrm{m}^{2}\right)$} & & & 0.594 & 0.553 \\
\hline & $25.42 \pm 2.39$ & $25.61 \pm 1.97$ & & \\
\hline \multirow[t]{2}{*}{ Gestational age (weeks) } & & & 0.432 & 0.665 \\
\hline & $31.13 \pm 3.16$ & $31.32 \pm 2.85$ & & \\
\hline \multirow[t]{2}{*}{ Mean arterial pressure $(\mathrm{mmHg})$} & & & 1.319 & 0.189 \\
\hline & $154.62 \pm 8.62$ & $152.85 \pm 9.74$ & & \\
\hline Fertility circumstance & & & 0.447 & 0.503 \\
\hline Unipara & $72(76.60)$ & $68(72.34)$ & & \\
\hline Multipara & $22(23.40)$ & $26(27.66)$ & & \\
\hline \multicolumn{2}{|l|}{ Place of residence } & & 0.223 & 0.636 \\
\hline City & $83(88.30)$ & $85(90.43)$ & & \\
\hline Countryside & $11(11.70)$ & $9(9.57)$ & & \\
\hline \multicolumn{2}{|l|}{ Ethnicity } & & 0.495 & 0.483 \\
\hline Han & $75(79.78)$ & $71(75.53)$ & & \\
\hline Minority & $19(20.21)$ & $23(24.47)$ & & \\
\hline \multicolumn{2}{|l|}{ Smoking history } & & 0.046 & 0.829 \\
\hline Yes & $13(13.83)$ & $12(12.77)$ & & \\
\hline No & $81(86.17)$ & $81(87.23)$ & & \\
\hline \multicolumn{2}{|l|}{ Drinking history } & & 0.367 & 0.544 \\
\hline With & $16(17.02)$ & $13(13.83)$ & & \\
\hline Without & $78(82.98)$ & $81(86.17)$ & & \\
\hline \multicolumn{2}{|l|}{ Exercise habit } & & 0.101 & 0.74 \\
\hline With & $27(28.72)$ & $29(30.85)$ & & \\
\hline Without & $67(71.28)$ & $65(69.15)$ & & \\
\hline \multirow[t]{2}{*}{ Proteinuria (g/24 h) } & & & 1.190 & 0.236 \\
\hline & $1.84 \pm 0.54$ & $1.94 \pm 0.61$ & & \\
\hline \multicolumn{2}{|l|}{ Degree of edema } & & 0.426 & 0.808 \\
\hline$+($ extended to the thigh $)$ & $24(25.53)$ & $28(29.79)$ & & \\
\hline$++($ extends to the vulva and abdomen $)$ & $54(57.45)$ & $51(54.26)$ & & \\
\hline$+++($ systemic edema or ascites $)$ & $16(17.02)$ & $15(15.96)$ & & \\
\hline
\end{tabular}

Table II. Comparison of clinical efficacy between the two groups [n (\%)].

\begin{tabular}{lccc}
\hline Curative effect & Research group $(\mathrm{n}=94)$ & Control group $(\mathrm{n}=94)$ & $\chi^{2}$ \\
\hline Significant effect & $74(78.72)$ & $55(58.51)$ & P-value \\
Effective & $16(17.02)$ & $23(24.47)$ & $16(17.02)$ \\
No effect & $4(4.26)$ & $78(82.97)$ & 8.057 \\
Overall response & $90(95.74)$ & 0.004 \\
\hline
\end{tabular}

group, which was significantly higher than that in the control group (82.97\%), $\mathrm{P}<0.05$ (Table II).

The eutocia rate of the study group was higher than that of the control group, and the cesarean section rate was lower than that of the control group, $\mathrm{P}<0.05$ (Table III).
There were a total of 9 adverse reactions in the study group, including 3 cases of postpartum hemorrhage, 1 case of eclampsia, 1 case of pulmonary edema, and 4 cases of cardiovascular disease. In the control group, there were 4 cases of eclampsia, 5 cases of postpartum hemorrhage, 2 cases of 
Table III. Comparison of labor outcomes between the two groups [n (\%)].

\begin{tabular}{lccc}
\hline Groups & Eutocia & Cesarean section & Premature birth \\
\hline Research group $(\mathrm{n}=94)$ & $75(79.78)$ & $13(13.82)$ & $6(6.38)$ \\
Control group $(\mathrm{n}=94)$ & $61(64.89)$ & $24(25.53)$ & $11(11.70)$ \\
$\chi^{2}$ & 5.21 & 4.072 & 1.617 \\
P-value & 0.02 & 0.043 & 0.203 \\
\hline
\end{tabular}

Table IV. Comparison of adverse reactions of patients in the two groups [n (\%)].

\begin{tabular}{lccc}
\hline & Research group $(\mathrm{n}=94)$ & Control group $(\mathrm{n}=94)$ & $\chi^{2}$ \\
\hline Eclampsia & $1(1.06)$ & $4(4.25)$ & P-value \\
Postpartum hemorrhage & $3(3.19)$ & $5(5.32)$ & $2(2.12)$ \\
Placental abruption & $0(0.00)$ & $3(3.19)$ & $5(5.32)$ \\
Pulmonary edema & $1(1.06)$ & $19(20.21)$ & 4.196 \\
Cardiovascular disease & $4(4.25)$ & & 0.040 \\
Total adverse reactions & $9(9.57)$ & & \\
\hline
\end{tabular}

A

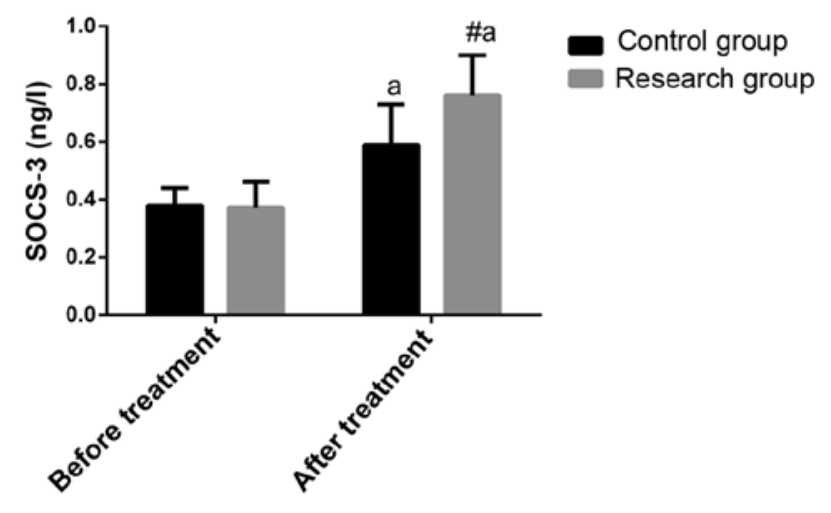

B

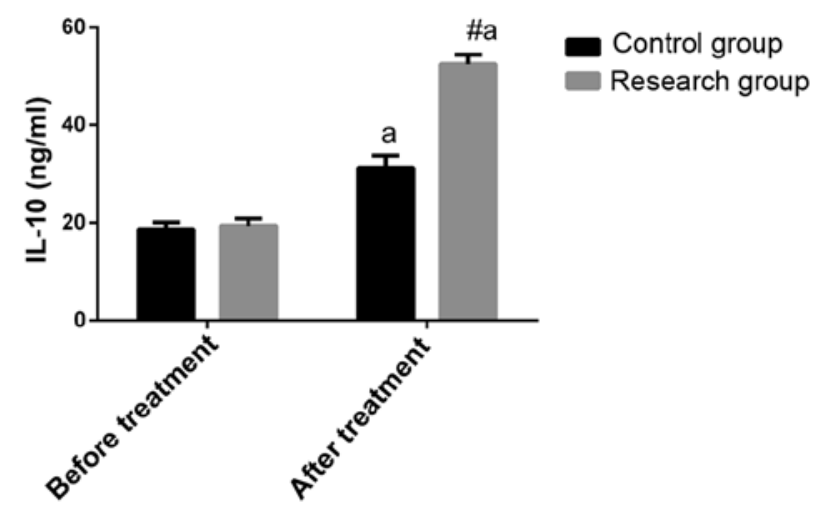

C

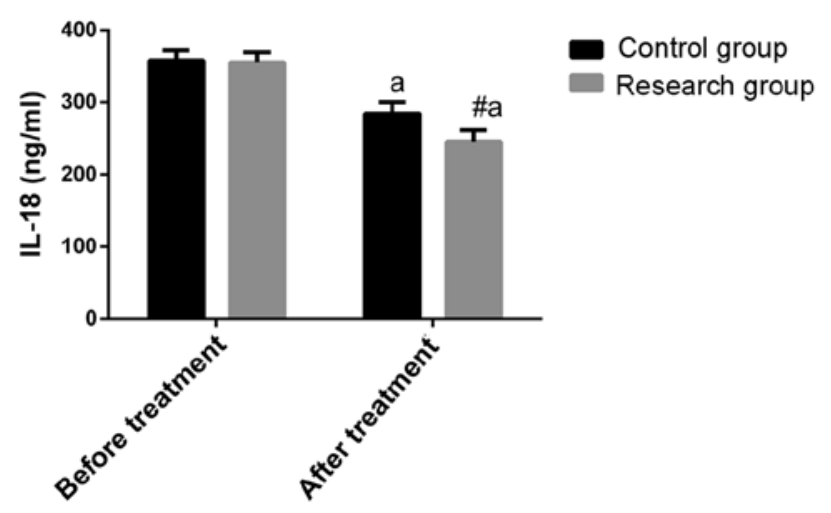

Figure 1. Curative effect of magnesium sulfate combined with nifedipine on pregnancy-induced hypertension and the effect of serum inflammatory factors. (A) Comparison of SOCS-3 levels before and after treatment between the two groups. Before treatment, there were no differences in SOCS-3 between the two groups $(\mathrm{P}>0.050)$. After treatment, SOCS-3 was significantly increased in both groups, and this in the study group was higher than that in the control group $(\mathrm{P}<0.050)$. (B) Comparison of IL-10 levels before and after treatment in both groups. Before treatment, there were no differences in IL-10 between the two groups $(\mathrm{P}>0.050)$. After treatment, IL-10 increased significantly in both groups, and this in the study group was higher than that in the control group $(\mathrm{P}<0.050)$. (C) Comparison of IL-18 levels before and after treatment in both groups. Before treatment, there were no differences in IL-18 between the two groups $(\mathrm{P}>0.050)$. After treatment, IL-18 decreased significantly in both groups, and this in the study group was lower than that in the control group $(\mathrm{P}<0.050)$ ${ }^{\#} \mathrm{P}<0.05$, compared with the control group; ${ }^{\text {a }}<<0.05$, compared with before treatment. SOCS-3, cytokine signaling-3; IL-10, interleukin-10; IL-18, interleukin-18. 


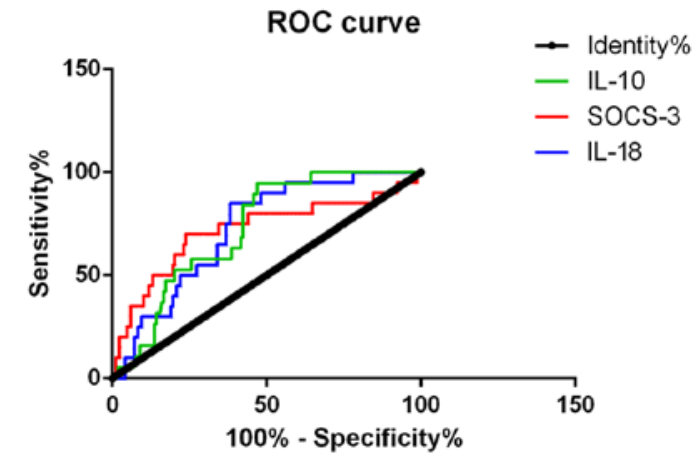

Figure 2. Predictive value of serum SOCS-3, IL-10 and IL-18 levels in patients with poor efficacy. SOCS-3, cytokine signaling-3; IL-10, interleukin-10; IL-18, interleukin-18.

placental abruption, 3 cases of pulmonary edema, and 5 cases of cardiovascular diseases, a total of 19 cases of adverse reactions occurred in the control group. The incidence of adverse reactions was significantly lower in the study group than that in the control group, $\mathrm{P}<0.05$ (Table IV).

Comparison of SOCS-3, IL-10 and IL-18 levels between the two groups before and after treatment. Before treatment, no differences were found in SOCS-3, IL-10 and IL-18 between the two groups $(\mathrm{P}>0.050)$. After treatment, the levels of SOCS-3 and IL-10 were significantly increased in the two groups, and they were higher in the study group than those in the control group $(\mathrm{P}<0.050)$. The IL-18 levels of the two groups were significantly decreased, and they were significantly lower in the study group than those in the control group $(\mathrm{P}<0.050$ (Fig. 1).

Predictive value of SOCS-3, IL-10 and IL-18 in patients with poor efficacy. All the patients were divided into good efficacy group $(n=168)$ and poor efficacy group $(n=20)$ in accordance with efficacy. According to the expression of SOCS-3, IL-10 and IL-18 in the two groups, ROC curve was drawn to analyze its predictive value for efficacy. The area under the curve (AUC) of SOCS-3 was 0.717 (95\% CI, 0.577-0.857). When the cut-off value was $<0.553 \mathrm{ng} / \mathrm{l}$, the optimal specificity was $76.19 \%$, the sensitivity was $70.00 \%$, and the Youden index was 46.19 . The AUC of IL-10 was 0.727 (95\% CI, 0.6356-0.817). When the cut-off value was $<48.06 \mathrm{ng} / \mathrm{ml}$, the optimal specificity was $52.98 \%$, the sensitivity was $94.74 \%$, and the Youden index was 47.71. The AUC of IL-18 curve was 0.725 (95\% CI, 0.630-0.821). When the cut-off value was $<269.4 \mathrm{ng} / \mathrm{ml}$, the optimal specificity was $61.90 \%$, the sensitivity was $85.00 \%$, and the Youden index was 46.90 (Fig. 2).

\section{Discussion}

Pregnancy-induced hypertension is an idiopathic disease and prone to occur in the third trimester of pregnancy $(20,21)$. Severe complications such as eclampsia appear in intensive cases $(22,23)$, which seriously affects the health and safety of pregnant women and infants. Early intervention can effectively prevent the occurrence of severe symptoms. The level of inflammatory factors in serum were studied to carry out early screening for pregnancy-induced hypertension and early drug intervention. In previous research, indexes such as IL-1, IL-2, IL-6, and PCT have been studied thoroughly, and were found with abnormal manifestations in many diseases (24-28), with low specificity.

Our study found that the total effective rate of clinical efficacy in the study group was $95.74 \%$, which was significantly higher than that in the control group (82.97\%), and the eutocia rate in the study group was higher than that in the control group, with better pregnancy results. It suggested that magnesium sulfate combined with nifedipine could effectively improve the clinical efficacy of in pregnancy-induced hypertension. Then the incidence of adverse reactions between the two groups were compared, and it was found that the incidence of adverse reactions in the study group was significantly lower than that in the control group, and there was only one case of adverse reactions (eclampsia) in the study group. This suggested that combination therapy reduces the incidence of adverse reactions, and some studies have shown that magnesium sulfate and nifedipine in the treatment of eclampsia does not increase the risk of serious magnesium-related effects, confirming the safety of combination therapy (29). In this study, we first analyzed the changes of SOCS-3, IL-10 and IL-18 levels in observation group and control group before and after treatment. It was found that SOCS-3, IL-10 levels in both groups increased significantly after treatment, and the level of IL-18 in both groups decreased significantly. The levels in the study group were significantly lower than those in the control group. This is consistent with the study of Chen et al (30) that IL-6 level in pregnant women with preeclampsia treated with magnesium sulfate combined with nifedipine decreased. SOCS-3 is an important molecule that regulates the function of trophoblasts during placental development (31). Clinical studies have shown that SOCS-3 is the target of miR-203 (32), and the decreased expression of SOCS-3 has been confirmed in the maternal vascular endothelium of preeclampsia. Wang et al (33) found that the expression of miR-203 was increased in the maternal vascular endothelium of women with preeclampsia, and the overexpression of miR-203 could down-regulate the expression of SOCS-3 in the endothelial cells, accompanied by inhibition of TNF- $\alpha$-induced increase in adhesion of neutrophils to endothelial cells. This suggests that the down-regulation of SOCS-3 and increased inflammatory response in the maternal vascular system may be the result of increased expression of miR-203 in preeclampsia. Subsequently, ROC curve analysis of the curative effect was performed and found that the AUC of the predictive value of SOCS-3, IL-10 and IL-18 in pregnancy-induced hypertension was $0.717,0.727$ and 0.725 , respectively. The best specificity was $76.19,52.98$ and $61.90 \%$, respectively, when the cut-off value was $<0.553 \mathrm{ng} / 1,48.06 \mathrm{ng} / \mathrm{ml}$ and $269.46 \mathrm{ng} / \mathrm{ml}$, and the sensitivity was $70.00,94.74$ and $85.00 \%$, respectively. This suggests that SOCO-3, IL-10 and IL-18 have certain value in predicting the efficacy of pregnancy-induced hypertension, and may be used as biomarkers to predict the clinical efficacy. We speculate that the combined detection of the above indicators in the future clinical practice can effectively determine the clinical efficacy of patients.

Although this study observed changes in inflammatory factors before and after treatment in the two groups of patients, we did not specifically analyze the treatment time and efficacy, 
leaving certain limitations. Thus, further studies are required on the relationship between SOCS-3, IL-10 and IL-18 and pregnancy-induced hypertension, to further corroborate the results of this study.

In conclusion, magnesium sulfate combined with nifedipine can significantly improve the prevalence of pregnancy-induced hypertension. The levels of SOCS-3, IL-10 and IL-18 in patients are related to the curative effect of pregnancy-induced hypertension, suggesting that they have important value in the treatment and monitoring of gestational hypertension.

\section{Acknowledgements}

Not applicable.

\section{Funding}

No funding was received.

\section{Availability of data and materials}

The datasets used and/or analyzed during the present study are available from the corresponding author on reasonable request.

\section{Authors' contributions}

FZ, FA and XD conceived and designed the study, and drafted the manuscript. FZ, FA, JW and XD collected, analyzed and interpreted the experimental data. FZ revised the manuscript for important intellectual content. All authors read and approved the final version of the manuscript.

\section{Ethics approval and consent to participate}

The study was approved by the Ethics Committee of The First People's Hospital of Yunnan Province (The Affiliated Hospital of Kunming University of Science and Technology). Signed informed consents were obtained from the patients and/or guardians.

\section{Patient consent for publication}

Not applicable.

\section{Competing interests}

The authors declare that they have no competing interests.

\section{References}

1. George JN and Amaresh A: Neonatal mortality and morbidity in pregnancy induced hypertension: A prospective observational study. J Evol Med Dent Sci 3: 5238-5246, 2014.

2. Zhuang C, Gao J, Liu J, Wang X, He J, Sun J, Liu X and Liao S: Risk factors and potential protective factors of pregnancy-induced hypertension in China: A cross-sectional study. J Clin Hypertens (Greenwich) 21: 618-623, 2019.

3. Kintiraki E, Papakatsika S, Kotronis G, Goulis DG and Kotsis V: Pregnancy-induced hypertension. Hormones (Athens) 14 211-223, 2015.

4. Andrews L and Patel N: Correlation of serum lactate dehydrogenase and pregnancy induced hypertension with its adverse outcomes. Int J Res Med Sci 4: 1347-1350, 2016.
5. Easterling TR: Pharmacological management of hypertension in pregnancy. Semin Perinatol 38: 487-495, 2014.

6. Chen $\mathrm{C}$ and Tao R: The impact of magnesium sulfate on pain control after laparoscopic cholecystectomy: A meta-analysis of randomized controlled studies. Surg Laparosc Endosc Percutan Tech 28: 349-353, 2018

7. Lamarca B,Brewer J and Wallace K: IL-6-induced pathophysiology during pre-eclampsia: Potential therapeutic role for magnesium sulfate? Int J Interferon Cytokine Mediat Res 2011: 59-64, 2011.

8. Alexander JM, McIntire DD, Leveno KJ and Cunningham FG: Selective magnesium sulfate prophylaxis for the prevention of eclampsia in women with gestational hypertension. Obstet Gynecol 108: 826-832, 2006.

9. Sharma KJ, Greene N and Kilpatrick SJ: Oral labetalol compared to oral nifedipine for postpartum hypertension: A randomized controlled trial. Hypertens Pregnancy 36: 44-47, 2017.

10. Webster LM, Myers JE, Nelson-Piercy C, Harding K, Cruickshank JK, Watt-Coote I, Khalil A, Wiesender C, Seed PT and Chappell LC: Labetalol versus nifedipine as antihypertensive treatment for chronic hypertension in pregnancy: A randomized controlled trial. Hypertension 70: 915-922, 2017.

11. Martínez-Jiménez C, Cruz-Angeles J, Videa M and Martínez LM: Co-amorphous simvastatin-nifedipine with enhanced solubility for possible use in combination therapy of hypertension and hypercholesterolemia. Molecules 23: 2161, 2018.

12. Wen $\mathrm{J}$ and Li X: Effect of magnesium sulfate combined with phentolamine and nifedipine for gestational hypertension and serum levels of LIF and apelin. J Coll Physicians Surg Pak 29: 231-234, 2019.

13. Sun Q, Xin F, Wen X, Lu C, Chen R and Ruan G: Protective effects of different kinds of filtered water on hypertensive mouse by suppressing oxidative stress and inflammation. Oxid Med Cell Longev 2018: 2917387, 2018

14. Niwa Y, Kanda H, Shikauchi Y, Saiura A, Matsubara K, Kitagawa T, Yamamoto J, Kubo T and Yoshikawa H: Methylation silencing of SOCS-3 promotes cell growth and migration by enhancing JAK/STAT and FAK signalings in human hepatocellular carcinoma. Oncogene 24: 6406-6417, 2005.

15. Wang Y, Lewis DF, Gu Y, Zhao S and Groome LJ: Elevated maternal soluble Gp130 and IL-6 levels and reduced Gp130 and SOCS-3 expressions in women complicated with preeclampsia. Hypertension 57: 336-342, 2011.

16. Yang GX, Sun Y, Tsuneyama K, Zhang W, Leung PS, He XS Ansari AA, Bowlus C, Ridgway WM and Gershwin ME: Endogenous interleukin-22 protects against inflammatory bowel disease but not autoimmune cholangitis in dominant negative form of transforming growth factor beta receptor type II mice. Clin Exp Immunol 185: 154-164, 2016.

17. Cubro H, Kashyap S, Nath MC, Ackerman AW and Garovic VD: The role of interleukin-10 in the pathophysiology of preeclampsia. Curr Hypertens Rep 20: 36, 2018.

18. Krishnan SM, Sobey CG, Latz E, Mansell A and Drummond GR: IL-1 $\beta$ and IL-18: Inflammatory markers or mediators of hypertension? Br J Pharmacol 171: 5589-5602, 2014.

19. Liu LS; Writing Group of 2010 Chinese Guidelines for the Management of Hypertension: 2010 Chinese guidelines for the management of hypertension. Zhonghua Xin Xue Guan Bing Za Zhi 39: 579-615, 2011 (In Chinese).

20. Świątkowska-Stodulska R, Kmieć P, Stefańska K and Sworczak K: Renin-Angiotensin-aldosterone system in the pathogenesis of pregnancy-induced hypertension. Exp Clin Endocrinol Diabetes 126: 362-366, 2018.

21. Cai G, Zhang B, Weng W, Yang L, Shi G, Xue S and Fu X: Associations of pregnancy-associated plasma protein-A level with essential hypertension and hypertensive disorders in pregnancy in Chinese population: A meta-analysis of 20 research studies involving 3332 individuals. BMJ Open 5: e008210, 2015.

22. Xu Q, Fan D, Li F and Zhang Z: Influence of serum HMW adiponectin level in patients with pregnancy-induced hypertension syndrome on the occurrence of eclampsia in secondary pregnancy. Exp Ther Med 14: 4972-4976, 2017.

23. Zhou Y, Chen ZH, Zhao XL, Qiao YH and Guo RX: Effect of serum high molecular weight adiponectin level on the occurrence of eclampsia during subsequent pregnancy in patients with primary pregnancy induced hypertension. Eur Rev Med Pharmacol Sci 21: 213-218, 2017.

24. Alves S, Churlaud G, Audrain M, Michaelsen-Preusse K, Fol R, Souchet B, Braudeau J, Korte M, Klatzmann D and Cartier N: Interleukin-2 improves amyloid pathology, synaptic failure and memory in Alzheimer's disease mice. Brain 140: 826-842, 2017. 
25. Parulekar AD, Kao CC, Diamant $Z$ and Hanania NA: Targeting the interleukin-4 and interleukin-13 pathways in severe asthma: Current knowledge and future needs. Curr Opin Pulm Med 24: 50-55, 2018.

26. Dinarello CA: Interleukin-1 mediated autoinflammation from heart disease to cancer. In: Textbook of Autoinflammation. Hashkes PJ, Laxer RM and Simon A (eds). Springer, Cham, pp711-725, 2019.

27. Ridker PM, Libby P, MacFadyen JG, Thuren T, Ballantyne C, Fonseca F, Koenig W, Shimokawa H, Everett BM and Glynn RJ: Modulation of the interleukin-6 signalling pathway and incidence rates of atherosclerotic events and all-cause mortality: Analyses from the Canakinumab Anti-Inflammatory Thrombosis Outcomes Study (CANTOS). Eur Heart J 39: 3499-3507, 2018.

28. Vijayan AL, Vanimaya, Ravindran S, Saikant R, Lakshmi S, Kartik R and Manoj G: Procalcitonin: A promising diagnostic marker for sepsis and antibiotic therapy. J Intensive Care 5: 51, 2017.

29. Magee LA, Miremadi S, Li J, Cheng C, Ensom MH, Carleton B, Côté AM and von Dadelszen P: Therapy with both magnesium sulfate and nifedipine does not increase the risk of serious magnesium-related maternal side effects in women with preeclampsia. Am J Obstet Gynecol 193: 153-163, 2005.
30. Chen Q, Zhao M, Guo F, Yin YX, Xiao JP, Stone PR and Chamley LW: The reduction of circulating levels of IL-6 in pregnant women with preeclampsia by magnesium sulphate and nifedipine: In vitro evidence for potential mechanisms. Placenta 36: 661-666, 2015.

31. Zhao S, Gu Y, Dong Q, Fan R and Wang Y: Altered interleukin-6 receptor, IL-6R and gp130, production and expression and decreased SOCS-3 expression in placentas from women with pre-eclampsia. Placenta 29: 1024-1028, 2008.

32. Wei T, Orfanidis K, Xu N, Janson P, Ståhle M, Pivarcsi A and Sonkoly E: The expression of microRNA-203 during human skin morphogenesis. Exp Dermatol 19: 854-856, 2010.

33. Wang Y, Dong Q, Gu Y and Groome LJ: Up-regulation of miR-203 expression induces endothelial inflammatory response: Potential role in preeclampsia. Am J Reprod Immunol 76: 482-490, 2016. 